\title{
Rekombinantes Milbenallergen auf dem Prüfstand
}

In der allergologischen Diagnostik sind rekombinante Allergene bereits Standard. In der spezifischen Immuntherapie ist es wohl nur eine Frage der Zeit, bis sie die natürlichen Extrakte ablösen werden. Ein hypoallergenes rekombinantes Hausstaubmilbenallergen musste sich jetzt im Tierversuch präklinisch bewähren.

\begin{abstract}
llergologen aus Österreich und Australien haben sich daran gemacht, eine rekombinante hypoallergene Kombinationsvakzine für die spezifische Immuntherapie von HausstaubmilbenAllergien zu entwickeln. Sie konzentrierten sich auf die beiden Dermatophagoides-pteronyssinus-Hauptallergene Der $\mathrm{p} 1$ und Der p 2, auf die über $95 \%$ der Hausstaubmilbenallergiker reagieren. Sie schleusten synthetische Gene, die für zwei Hybridproteine kodieren, in Escherichia-coli-Bakterien ein. Die von den Bakterien gebildeten Proteine reinigten die Forscher chromatografisch auf. Beide Proteine sind aus Der-p-1- und Derp-2-Fragmenten aufgebaut, das eine beinhaltet zusätzlich Cystein (rekombinates $[\mathrm{r}]$ Der $\mathrm{p} 2 / 1 \mathrm{C}$ ) das andere nicht (rDer p 2/1S).
\end{abstract}

Die allergene Potenz wurden anhand der IgE-Reaktivität und der Basophilenaktivierung bestimmt. Inwieweit genügend T-Zell-Epitope vorhanden waren, überprüften die Forscher mithilfe mononukleärer Zellen aus dem Blut von Milbenallergikern. Die allergene Potenz der Hybridmoleküle sowie deren Fähigkeit, die Synthese allergenspezifischer IgG zu induzieren, testeten sie an Mäusen und Kaninchen.

Die Ergebnisse überzeugten die Forscher: Die geimpften E.-coli-Stämme exprimierten rDer $\mathrm{p} 2 / 1 \mathrm{C}$ und rDer $\mathrm{p} 2 / 1 \mathrm{~S}$ in großen Mengen als lösliches, gefaltetes Protein. Beide Hypoallergene lösten weder eine relevante IgE-Reaktivität, noch eine allergische Entzündung oder eine allergische Antwort aus. Sie induzierten beide eine ähnlich starke T-Zell-

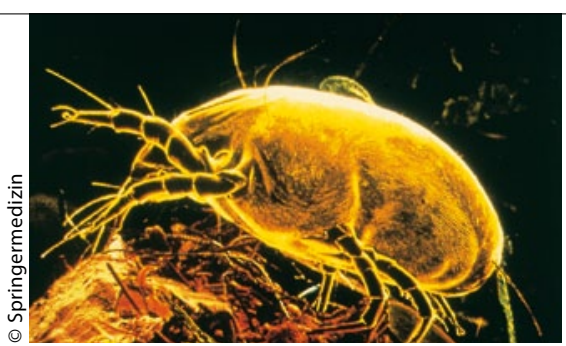

Proliferation wie die natürlichen Allergene. Bei der Immunisierung von Kaninchen mit diesen Hypoallergenen konnten IgG-Antikörper produziert werden, die die IgE-Reaktivität auf Der p 1 und Der p 2 unterdrückten. Dabei schnitt das cysteinfreie Molekül etwas besser $a b$ als das cysteinhaltige.

Fazit: Aufgrund der präklinischen Charakterisierung beurteilen die Autoren die rekombinanten Hypoallergene als sicher und wirksam für die Therapie der Hausstaubmilbenallergie. Für die klinische Testung wird jetzt das cysteinfreie rDer p 2/1S-Molekül verwendet: Zum einen weil nur rDer $\mathrm{p} 2 / 1 \mathrm{~S}$ monomer vorliegt, zum anderen weil es keine relevante Basophilenreaktion auslöst und die allergische Reaktion stärker unterdrückt als rDer p 2/1C.

Dr. Dagmar Kraus

Chen K-W et al. Hypoallergenic Der p 1/Der $\mathrm{p} 2$ combination vaccines for immunotherapy of house dust mite allergy. J Allergy Clin Immunol 2012; 130: 435-43

\section{Asthma oder nicht? Das IgE-Profil weiß es}

\section{Eine allergische Sensibilisierung - nachgewiesen durch das Vorhandensein von spezifischen IgE im Serum - ist ein wichtiger Risikofaktor für die Entwicklung eines Asthma bronchiale. Schwedische Mediziner haben untersucht, welche Allergene dabei bevorzugt eine Rolle spielen.}

$\mathrm{M}$ ithilfe von Microarrays analysierten die Forscher das IgE-Profil von 96 Asthmapatienten und 371 Kontrollpersonen ohne Asthma aus dem European Community Respiratory Health Survey II. Von 103 untersuchten Allergenkomponenten wurden 78 bei mindestens einem Patienten positiv getestet. IgE-Antikörper gegen mindestens eines der Allergene ließen sich bei 141 (38,0\%) der asthmafreien und bei $70(72,9 \%)$ der asthmakranken Teilnehmer nachweisen. Bei Asthmapatienten fand sich insbesondere signifikant häufiger IgE gegen pflanzliche Nahrungsmittel, Gräser- und Baumpollen, Felltiere, Schimmel und Latex.
Wurden die getesteten Allergene in vier Gruppen eingeteilt - Nahrung, Pollen, perenniale Allergene (z.B. Schimmel, Tiere) und andere (z. B. Latex, Kakerlaken) -, war die Sensibilisierung gegen perenniale Aeroallergene am stärksten mit Asthma assoziiert (Odds Ratio [OR]: 5,6). Als weiterer unabhängiger Risikofaktor erwies sich die Anwesenheit von IgE gegen Pollen (OR: 2,2). Der Nachweis von IgE-Antikörpern gegen ganzjährige Allergene und Pollen korrelierte außerdem mit steroidsensitiven entzündlichen Prozessen in den Atemwegen. Eine Sensibilisierung gegen kontinuierlich vorhandene Allergene war der einzige unabhängige Risikofaktor für eine bronchiale Hyperreagibilität im Metacholin-Test. Wenn bei pollensensibilisierten Personen eine Sensibilisierung gegen Nahrungsmittel hinzukam, steigerte dies das Asthmarisiko. Das höchste Risiko für Asthma (OR: 18,6) sowie Atemwegsentzündung und bronchiale Überempfindlichkeit bestand bei Personen, die gleichzeitig IgE-Antikörper gegen ganzjährige Allergene und Pollen sowie Nahrungsmittel produzierten.

Fazit: Mehrfache IgE- Sensibilisierungen gehen mit Atemwegsentzündung, bronchialer Hyperreagibilität und einem erhöhten Asthmarisiko einher. Durch eine Sensibilisierung gegen Nahrungsmittelallergene steigt das Asthmarisiko von Personen, die gegen Pollen sensibilisiert sind, zusätzlich.

Dr. Beate Schumacher

Patelis A et al. Population-based study of multiplexed IgE sensitization in relation to asthma, exhaled nitric oxide, and bronchial responsiveness. J Allergy Clin Immunol 2012; 130: 397-402 